\title{
Visfatin promotes osteosarcoma cell migration and invasion via induction of epithelial-mesenchymal transition
}

\author{
GONG CHENG ${ }^{1,2^{*}}$, CHANGYING LIU ${ }^{3 *}$, XIUJIANG SUN ${ }^{2}$, LEI ZHANG ${ }^{4}$, \\ LIFANG LIU ${ }^{5}$, JUN OUYANG ${ }^{1}$ and $\mathrm{BO} \mathrm{LI}^{5}$ \\ ${ }^{1}$ Department of Anatomy, Southern Medical University, Guangzhou, Guangdong; ${ }^{2}$ Department of Sports Medicine, \\ Yantaishan Hospital, Yantai, Shandong; ${ }^{3}$ Department of Orthopedics, People's Hospital of Linyi City, \\ Linyi, Shandong; ${ }^{4}$ Department of Gastroenterology, Central Hospital of Zibo, Zibo, Shandong; \\ ${ }^{5}$ Department of Cardiology, Central Hospital of Zibo, Zibo, Shandong, P.R. China
}

Received January 17, 2015; Accepted April 2, 2015

DOI: $10.3892 /$ or.2015.4053

\begin{abstract}
Visfatin is considered to be a biomarker in various types of cancers. However, no evidence has been reported for the direct effect of visfatin on osteosarcoma cell metastasis. The aims of the present study were to investigate the influence of visfatin on the migration and invasion of osteosarcoma cells and clarify the underlying mechanism. The expression levels of epithelial-mesenchymal transition (EMT) markers, as well as the transcriptional factor Snail-1, were first detected at both the protein and mRNA levels in U2OS osteosarcoma cells after stimulation of visfatin. Then the expression of NF- $\kappa \mathrm{B}(\mathrm{p} 65)$ was detected by western blot analysis, and siRNA of Snail-1 and inhibitor of $N F-\kappa B$ were used to investigate the effect of visfatin. Finally, migration and invasion of the cells were detected respectively by scratch wound healing and Transwell assays. Visfatin downregulated E-cadherin and upregulated $\mathrm{N}$-cadherin in concentration- and time-dependent manners at the protein and mRNA levels. The expression of Snail-1 was also upregulated. Moreover, visfatin also promoted the nuclear translocation of the NF- $\mathrm{BB}$ pathway. Administration of siRNA of Snail-1 and the inhibitor BAY11-7082 validated the roles of Snail-1 and NF- $\kappa$ B in the visfatin-induced regulation of EMT markers. Migration and invasion of U2OS osteosarcoma cells were promoted following the application of visfatin. These results demonstrated that visfatin enhances the migration and invasion of osteosarcoma cells via the NF- $\kappa \mathrm{B} /$ Snail-1/EMT pathway.
\end{abstract}

Correspondence to: Dr Bo Li, Department of Cardiology, Central Hospital of Zibo, No. 54 Gong Qing Tuan Xi Road, Zibo, Shandong 255036, P.R. China

E-mail: libosubmit@163.com

Professor Jun Ouyang, Department of Anatomy, Guangdong Provincial Medical Biomechanical Key Laboratory, Southern Medical University, Guangzhou, Guangdong 510515, P.R. China

E-mail: jouyang@126.com

${ }^{*}$ Contributed equally

Key words: visfatin, NF-кB, Snail-1, epithelial-mesenchymal transition

\section{Introduction}

Osteosarcoma is the most common malignant bone tumor, with high morbidity and lethality in young adults and adolescents. Although intensive chemotherapy, radiotherapy, as well as aggressive surgical procedures, have been combined and applied for the treatment of patients with osteosarcoma, only $60 \%$ achieve 5-year survival. What is striking is that $~ 80 \%$ of these patients have clinically detectable metastatic disease at the time of diagnosis $(1,2)$. This disappointing current situation warrants the urgent need to identify the underlying molecular signaling mechanisms involved in osteosarcoma carcinogenesis and metastasis.

Visfatin, initially found in visceral fat, also known as nicotinamide phosphoribosyltransferase (Nampt) and pre-Bcell colony-enhancing factor (PBEF), plays an important role in a variety of metabolic and stress responses, such as NAD biosynthesis, and exhibits proliferative, anti-apoptotic, proinflammatory and pro-angiogenic properties (3). Recently, the effects of visfatin on carcinogenesis and its use as a chemotherapeutic target have attracted our attention. Visfatin was found to be overexpressed in colorectal cancer (4), and its overexpression predicted a poor prognosis and decreased response to doxorubicin therapy in breast cancer (5). These data indicate that the expression and activity of visfatin may become novel therapeutic strategy targets for cancer patients. However, to date, only a few studies exist that concern the effect of visfatin on the clinical outcome of osteosarcoma patients, and even less studies have researched the potential mechanisms (6).

Epithelial-mesenchymal transition (EMT), whose function was primarily found in the process of embryogenesis (7), has been characterized by promoting downregulation of intercellular cohesion of cancer cells, elevated rate of cancer cell migration and invasion, and increased resistance to apoptosis even under chemotherapeutics (8). The most important hallmarks of EMT are decreased expression of the epithelial molecule E-cadherin and increased expression of mesenchymal marker N-cadherin (9). Previous studies have demonstrated that EMT significantly induces cancer cells to disseminate from the tumor mass leading to distant metastasis. 
However, there is still no evidence to date on the direct effect of visfatin on osteosarcoma cell migration and invasion. Thus, in view of the above, in the present study, we aimed to evaluate whether osteosarcoma cell migration and invasion are influenced by visfatin through promotion of EMT. Finally, the mechanism of EMT promotion in osteosarcoma cells was also investigated, thus, offering a potential improvement in osteosarcoma treatment management.

\section{Materials and methods}

Reagents. Recombinant human visfatin and BAY11-7082, a selective inhibitor of $\mathrm{NF}-\kappa \mathrm{B}$, were both purchased from Sigma-Aldrich (St. Louis, MO, USA). Rabbit monoclonal antibody to E-cadherin was purchased from Abcam (Cambridge, UK). Rabbit polyclonal antibodies to N-cadherin, Snail-1 and GAPDH were also purchased from Abcam. Rabbit anti-mouse antibody for NF- $\kappa$ B (P65) was purchased from Cell Signaling Technology Inc. (Danvers, MA, USA). Snail-1 siRNA was purchased from Santa Cruz Biotechnology, Inc. (Dallas, TX, USA).

Cell culture. The U2OS osteosarcoma cell line was purchased from the American Type Culture Collection (ATCC; Manassas, VA, USA). Dulbecco's modified Eagle's medium (DMEM; HyClone, Logan, UT, USA) supplemented with $10 \%$ fetal bovine serum (FBS; HyClone) was used to culture the U2OS cells. The U2OS cells were grown in a $37^{\circ} \mathrm{C}$ incubator with $5 \% \mathrm{CO}_{2}$. To determine the effect of visfatin on the expression of EMT markers, the U2OS cells were maintained in DMEM supplemented with $1 \%$ FBS for $12 \mathrm{~h}$ and subsequently cultured in the absence or presence of visfatin or other reagents for the specified times.

Migration assay. Cell migration was studied using a scratch wound healing assay. The U2OS cells were cultured in 6 -well plates (1×10\% a confluent monolayer. Straight scratches of the same width were made in monolayers of the U2OS cells with a pipette tip. After incubation with the reagents for a specified time, photo images were captured to measure the wound healing under a microscope.

Invision assay. Cell invasion was studied using a Matrigelcoated Transwell assay. Modified Boyden chambers with $8-\mu \mathrm{m}$ pore filter inserts were coated with Matrigel (50 $\mu \mathrm{g} / \mathrm{well}$; BD Biosciences, San Jose, CA, USA). The U2OS cells were cultured in 24-well plates, and the upper chamber contained cells in DMEM plus 1\% FBS, while the lower chamber contained DMEM plus $10 \%$ FBS. The cells $\left(1 \times 10^{5}\right.$ cells/well $)$ were re-suspended in the upper chamber at $37^{\circ} \mathrm{C}$ and $5 \% \mathrm{CO}_{2}$. After a 24-h incubation, the cells that had invaded onto the lower surface of the Matrigel-coated membrane were fixed with methanol for $30 \mathrm{~min}$ and stained with hexamethylpararosaniline, while the cells that remained on the upper surface were wiped away.

Western blot analysis. After stimulation with visfatin and other reagents, the U2OS cells were collected and the protein was extracted in ice-cold RIPA buffer (Beyotime Institute of Biotechnology, Nantong, Jiangsu, China) with $1 \mathrm{mM}$ phenylmethyl-sulfonyl fluoride (PMSF; Beyotime Institute of Biotechnology) for $30 \mathrm{~min}$. Concentration of the extracted protein was measured using the BCA protein assay kit (Beyotime Institute of Biotechnology). Cell lysates were electrophoresed on a $10 \%$ SDS polyacrylamide gel and transferred onto nitrocellulose (NC) membranes (Millipore, Merck KGaA, Darmstadt, Germany). The blots were blocked and incubated with primary antibodies overnight followed by incubation with the secondary antibodies for $2 \mathrm{~h}$ at room temperature. Finally, the blots were visualized with an electrochemiluminescence (ECL) detection system (Millipore).

Real-time quantitative PCR ( $P$ PCR) analysis. Total RNA was isolated and extracted using TRIzol reagent (Invitrogen) according to the manufacturer's directions from the cells that were cultured following the specified experimental programs. The concentration of total RNA was quantified by spectrophotometry. The RNA samples were reverse-transcribed into cDNA using M-MLV (Moloney murine leukemia virus) reverse transcriptase system (Fermentas, Shenzhen, China) (10). Total cDNA was amplified and detected using LightCycler FastStart DNA Master SYBR-Green I (Takara Biotechnology, Dalian, China). 18s was chosen as the reference gene, and the primer sequences for real-time PCR analyses were: E-cadherin forward primer, 5'-ACCAGAATAAAGACCAAGTGACCA-3' and reverse primer, 5'-AGCAAGAGCAGCAGAATCAG AAT-3' (11); N-cadherin forward primer, 5'-CACTGCTCA GGACCCAGAT-3' and reverse primer, 5'-TAAGCCGAGT GATGGTCC-3' (12); Snail forward primer, 5'-TTGGATAC AGCTGCTTTGAG-3' and reverse primer, 5'-ATTGCATA GTTAGTCACACCTC-3' (11); 18S forward primer, 5'-CTT AGTTGGTGGAGCGATTTG-3' and reverse primer, 5'-GCT GAACGCCACTTGTCC-3' (13).

Statistical analysis. Data in the present study were evaluated with Predictive Analytic Software (PASW) Statistics 18.0 (SPSS, Inc., Chicago, IL, USA). The normally distributed data were analyzed by one-way ANOVA, while the nonparametric variables were analyzed by the Mann-Whitney $\mathrm{U}$ test. Statistical significance was confirmed at a probability value $(\mathrm{P})<0.05$. Each assay was performed in triplicate.

\section{Results}

Effects of visfatin on expression of EMT markers in the osteosarcoma cells. It is well known that cancer metastasis involves multiple steps, among which the acquisition of invasiveness through EMT has been well established and illustrated. Meanwhile, the loss of the epithelial marker E-cadherin and gain of mesenchymal marker $\mathrm{N}$-cadherin have been both confirmed as the most important hallmarks of EMT (9). We thus examined the effects of visfatin on expression of EMT markers in the osteosarcoma cells with western blot analysis and real-time PCR. The osteosarcoma cells were cultured with various concentrations of visfatin $(0,5,50$ and $500 \mathrm{ng} /$ $\mathrm{ml}$ ) respectively, for $24 \mathrm{~h}$. As shown in Fig. 1A and C, visfatin concentration-dependently decreased expression of the epithelial marker E-cadherin and enhanced the expression of mesenchymal marker $\mathrm{N}$-cadherin in the osteosarcoma cells. 
A

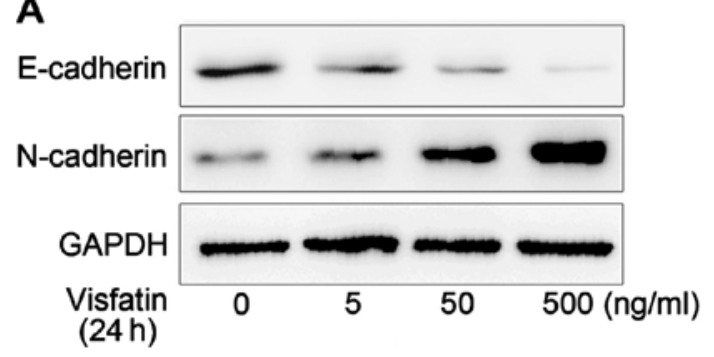

C

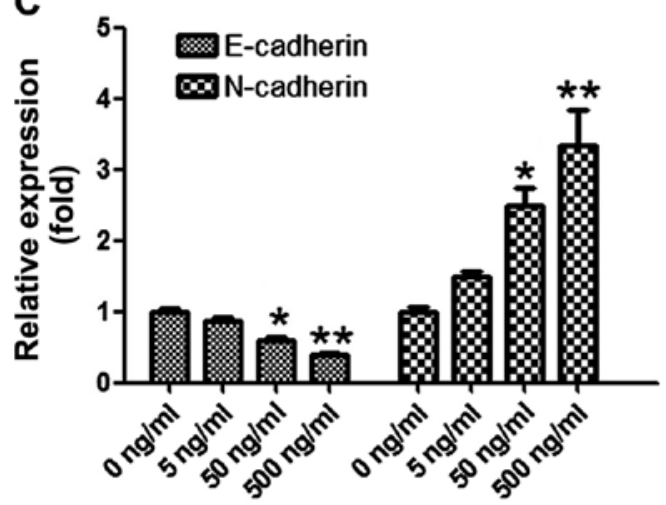

E

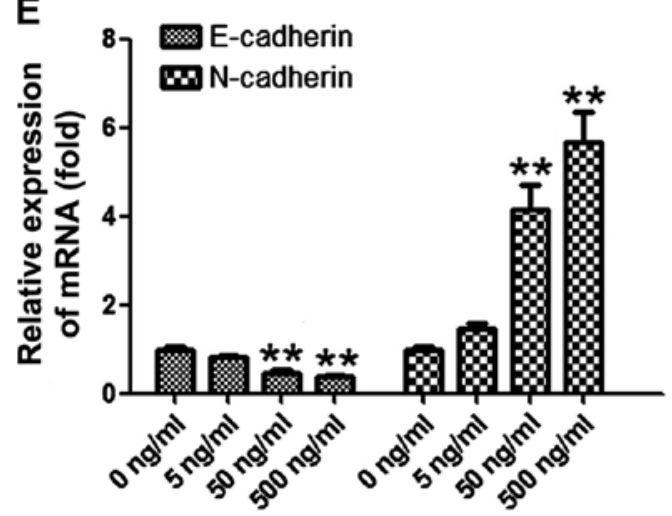

B

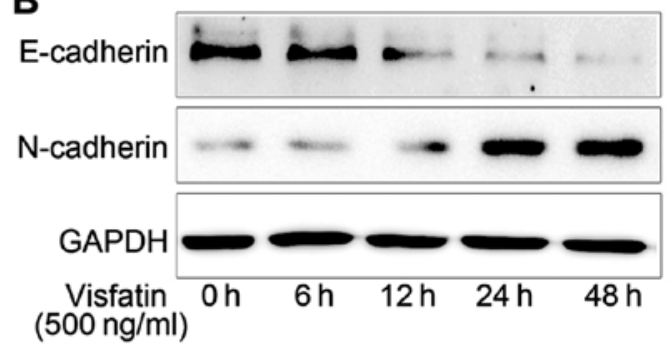

D

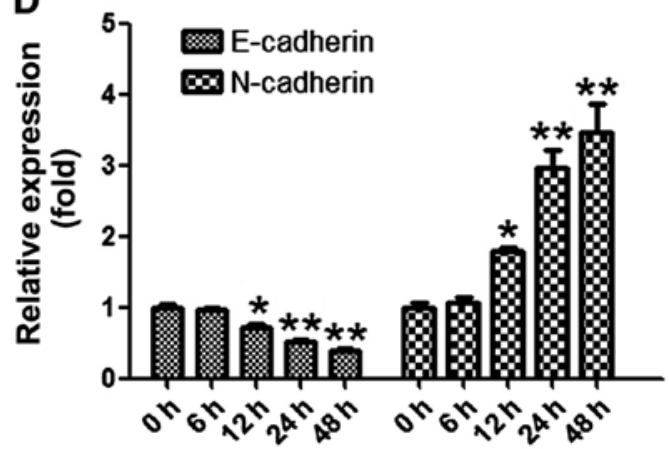

F

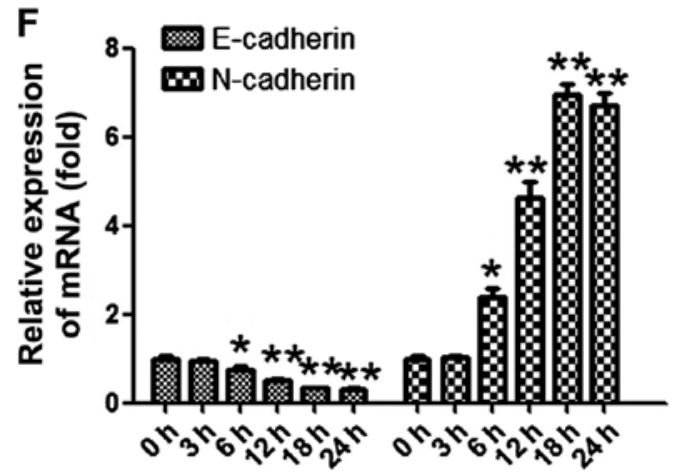

Figure 1. Visfatin decreases the expression of E-cadherin and increasesthe expression of N-cadherin in U2OS osteosarcoma cells. (A, C and E) U2OS osteosarcoma cells were treated with visfatin at various concentrations $(0,5,50$ and $500 \mathrm{ng} / \mathrm{ml})$ for $24 \mathrm{~h}$. (A and C) Western blot assay was used to assess the changes in E-cadherin and N-cadherin protein expression levels. (E) RT-PCR was also used to detect the mRNA expression levels of E-cadherin and N-cadherin. (B, D and F) U2OS osteosarcoma cells were treated with visfatin at the concentration of $500 \mathrm{ng} / \mathrm{ml}$ for various times $(0,6,12,24$ and $48 \mathrm{~h}$ ). (B and D) Western blot assay was used to assess the changes in E-cadherin and N-cadherin protein expression levels. (F) RT-PCR was also used to detect the mRNA expressions of E-cadherin and N-cadherin. ${ }^{*} \mathrm{P}<0.05$ vs. the control group; ${ }^{* *} \mathrm{P}<0.01$ vs. the control group. Data shown are means \pm SEM from three independent experiments in duplicate.

Then, real-time PCR was performed and the relative expression of the mRNA levels of E-cadherin and N-cadherin were assessed, and the results demonstrated the same tendency as the expression levels at the protein level (Fig. 1E). Subsequently, visfatin of $500 \mathrm{ng} / \mathrm{ml}$ was added to the osteosarcoma cells and cultured for various times $(0,6,12,24$ and $48 \mathrm{~h})$. As shown in Fig. $1 \mathrm{~B}$ and D, visfatin time-dependently decreased the expression of E-cadherin and enhanced expression of $\mathrm{N}$-cadherin in the osteosarcoma cells, reaching peak activity at $48 \mathrm{~h}$. Finally, we also examined the mRNA expression of E-cadherin and $\mathrm{N}$-cadherin after various culture times $(0,3,6,12,18$ and $24 \mathrm{~h})$. Similar results as the protein level were obtained. The results above indicated that visfatin altered the expression of EMT markers in a concentration- and time-dependent manner.
Visfatin affects EMT via regulation of the expression of Snail. It has been revealed that EMT is affected by a variety of regulatory networks and is mainly triggered by a series of transcription factors, such as Snail-1, Slug, Twist, Zeb1 and SIP1. Among these factors, Snail-1 has been the most extensively studied and has been found to play a pivotal role in the process (14). Thus, we investigated the effects of visfatin on the expression of Snail-1 and its subsequent effects on EMT.

Visfatin of $500 \mathrm{ng} / \mathrm{ml}$ was selected to stimulate osteosarcoma cells for various times $(0,3,6,9$ and $12 \mathrm{~h})$. As shown in Fig. 2A, visfatin significantly promoted the expression of Snail- 1 along with the increase in the stimulation time, reaching a peak at $12 \mathrm{~h}$. Subsequently, real-time PCR was performed to examine the relative expression of Snail-1, and the results were 

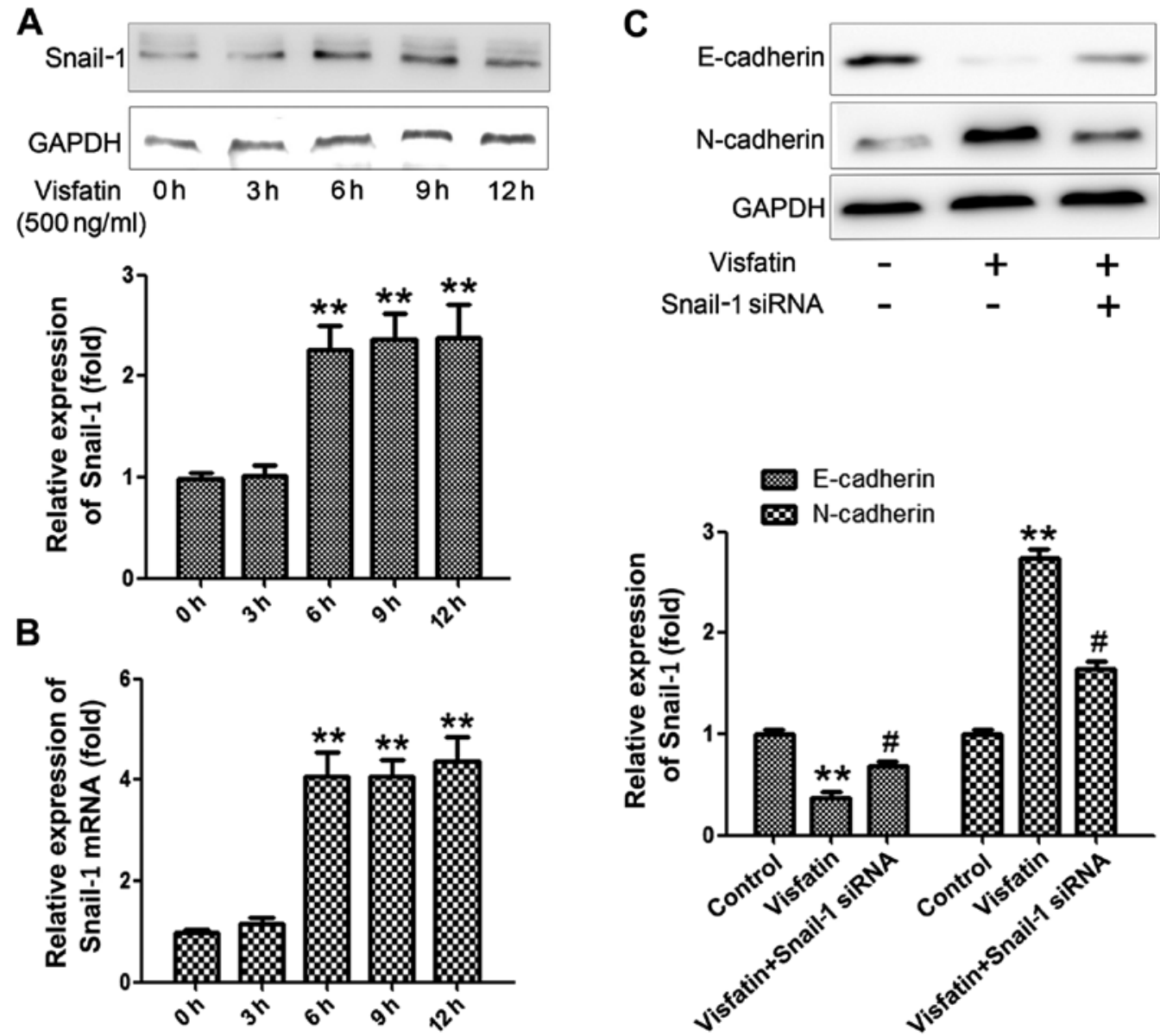

Figure 2. Snail-1 mediates the regulation of EMT markers. (A and B) U2OS osteosarcoma cells were treated with visfatin at the concentration of $500 \mathrm{ng} / \mathrm{ml}$ for various times $(0,3,6,9$ and $12 \mathrm{~h}$ ). (A) Western blot assay and (B) RT-PCR assay were both used to assess the changes in Snail-1 protein expression. (C) Western blot analysis showed that Snail-1 siRNA significantly reversed the effects of visfatin on E-cadherin and N-cadherin expression. "P<0.05 vs. the control group; ${ }^{* *} \mathrm{P}<0.01$ vs. the control group; ${ }^{*} \mathrm{P}<0.05$ vs. the visfatin group. Data shown are means \pm SEM from three independent experiments in duplicate.
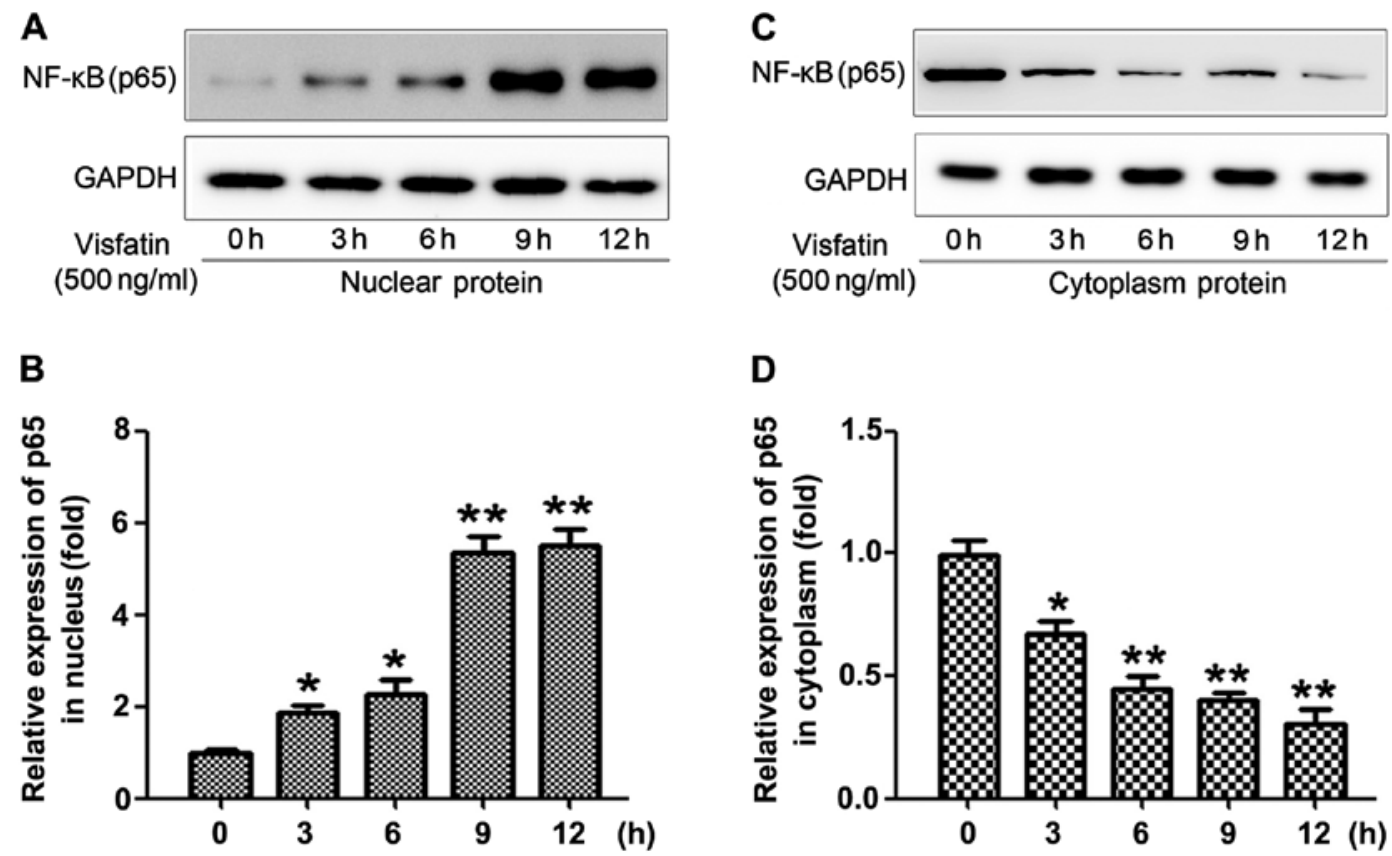

Figure 3. Expression of NF- $\kappa$ B is influenced by visfatin. (A and B) Treatment with visfatin $(500 \mathrm{ng} / \mathrm{ml})$ for various times $(0,3,6,9$ and $12 \mathrm{~h})$ time-dependently increased the expression of p65 in the nucleus of osteosarcoma cells. (C and D) Treatment with visfatin $(500 \mathrm{ng} / \mathrm{ml})$ for various times $(0,3,6,9$ and $12 \mathrm{~h})$ time-dependently decreased the expression of $\mathrm{p} 65$ in the cytosol. ${ }^{*} \mathrm{P}<0.05$ vs. the control group; ${ }^{* *} \mathrm{P}<0.01$ vs. the control group. Data shown are means $\pm \mathrm{SEM}$ from three independent experiments in duplicate. 
A

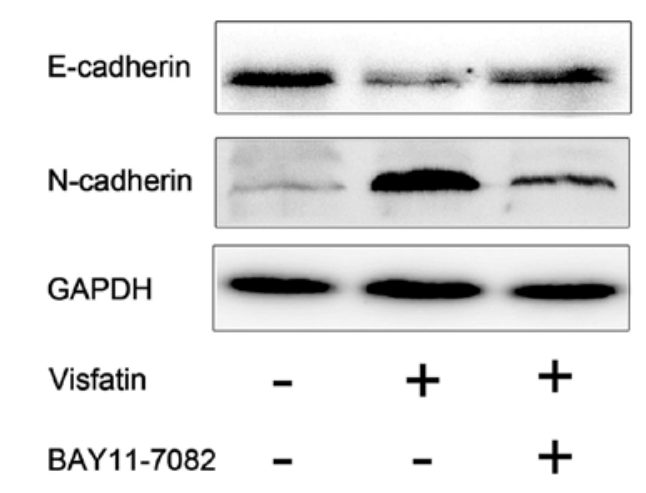

C

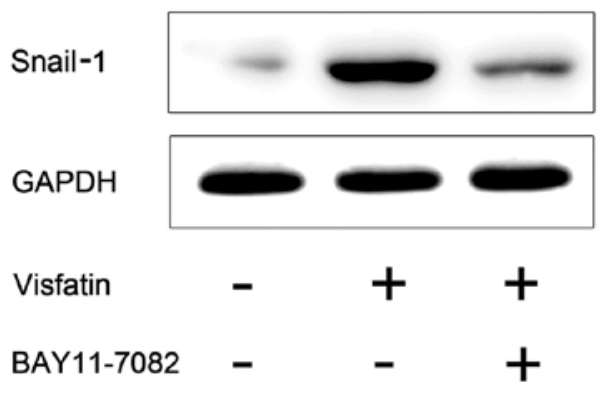

B

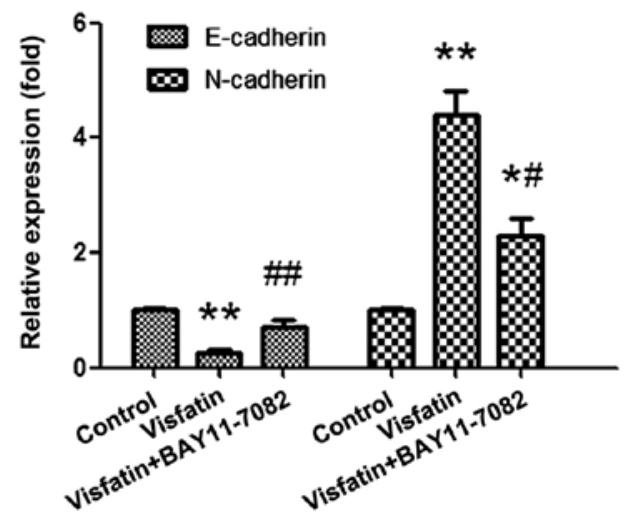

D

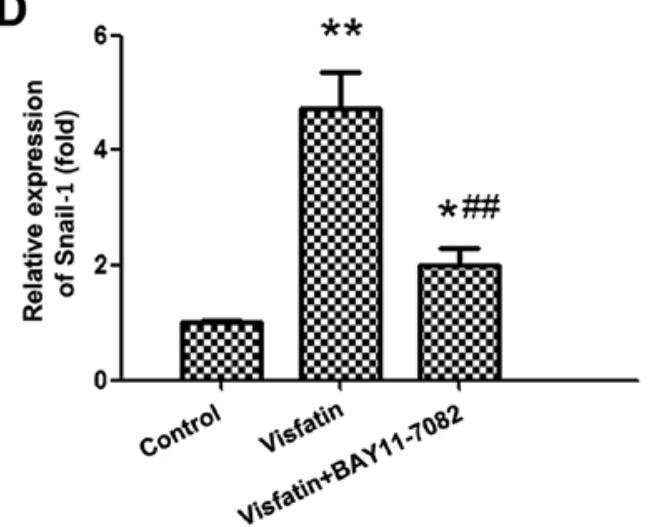

Figure 4. NF-kB mediates the regulation of EMT markers and Snail-1. (A and B) Western blot analysis showed that BAY11-7082 (20 $\mu$ M) significantly reversed the effects of visfatin on E-cadherin and N-cadherin expression. (C and D) Western blot analysis showed that BAY11-7082 (20 $\mu$ M) significantly reversed the effects of visfatin on Snail-1. ${ }^{*} \mathrm{P}<0.05$ vs. the control group; ${ }^{* *} \mathrm{P}<0.01$ vs. the control group; ${ }^{*} \mathrm{P}<0.05$ vs. the visfatin group; ${ }^{\# \#} \mathrm{P}<0.01$ vs. the visfatin group. Data shown are means \pm SEM from three independent experiments in duplicate.

similar with those at the protein level (Fig. 2B). Subsequently, in order to ascertain the compulsory role of Snail-1 in promoting EMT, the cells were pre-treated with siRNA of Snail-1 for $2 \mathrm{~h}$ before the stimulation of visfatin. As shown in Fig. 2C, pretreatment with the siRNA of Snail-1 significantly abrogated the promotive effect of visfatin on E-cadherin and the inhibitory effect on $\mathrm{N}$-cadherin. The results above confirmed that Snail-1 is a key regulator of EMT and visfatin affects EMT via regulation of the expression of Snail-1.

Visfatin induces $N F-\kappa B$ nuclear translocation. It has been reported that $\mathrm{NF}-\kappa \mathrm{B}$ is a key transcriptional signaling molecule that mediates the expression of many downstream genes. In previous studies, the expression of $\mathrm{NF}-\kappa \mathrm{B}$ and its nuclear translocation were proven to be involved in the invasion of osteosarcoma cells $(15,16)$. Thus, in the present study, in order to determine the effects of visfatin on the expression and nuclear translocation of $\mathrm{NF}-\kappa \mathrm{B}$, we respectively extracted and measured the phosphorylation levels of p65 in the nucleus and in the cytoplasm after various stimulation times $(0,3,6,9$ and $12 \mathrm{~h}$ ). As shown in Fig. 3A and B, the results demonstrated that visfatin significantly increased the expression of p65 in the nucleus and simultaneously decrease its expression in the cytoplasm (Fig. 3C and D). In addition, we observed that only a 3 -h stimulation by visfatin could significantly transfer p65 from the cytoplasm to the nucleus.
$N F-\kappa B$ mediates visfatin-induced expression of EMT markers. In order to further determine the molecular mechanisms underlying visfatin-induced promotion and suppression of EMT markers, we chose $500 \mathrm{ng} / \mathrm{ml}$ of visfatin to stimulate the osteosarcoma cells for $24 \mathrm{~h}$ and pre-treated the cells with BAY11-7082 $(20 \mu \mathrm{M})$ for $2 \mathrm{~h}$. As shown in Fig. 4A, pretreatment with the NF- $\mathrm{B}$ inhibitor BAY11-7082 significantly abrogated the effects of visfatin on E-cadherin and N-cadherin. Subsequently, the expression of Snail-1 was examined following the same treatments, and a similar tendency was obtained. These results indicate that $\mathrm{NF}-\kappa \mathrm{B}$ is a key signaling molecule that mediates the expression of Snail-1 and EMT.

Visfatin promotes the migration and invasion of osteosarcoma cells mainly through the Snail-1 and $N F-\kappa B$ signaling pathways. Wound healing and Transwell assays were respectively applied to evaluate the migration and invasion of osteosarcoma cells. Osteosarcoma cells were treated with various concentrations of visfatin $(0,5,50$ and $500 \mathrm{ng} / \mathrm{ml})$ for $24 \mathrm{~h}$. As shown in Fig. 5A, the results of the wound healing assay demonstrated that healing over the scratch was increased gradually in a concentration-dependent manner after treatment with visfatin. Moreover, as shown in Fig. 5C, a Transwell assay exhibited that the number of invading cells transferred from the upper surfaces to the lower surfaces were also prominently promoted by visfatin in a similar trend. Next, in order to 
A

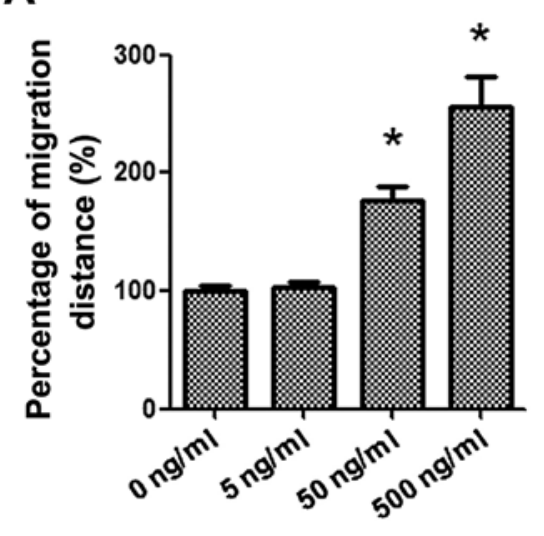

C

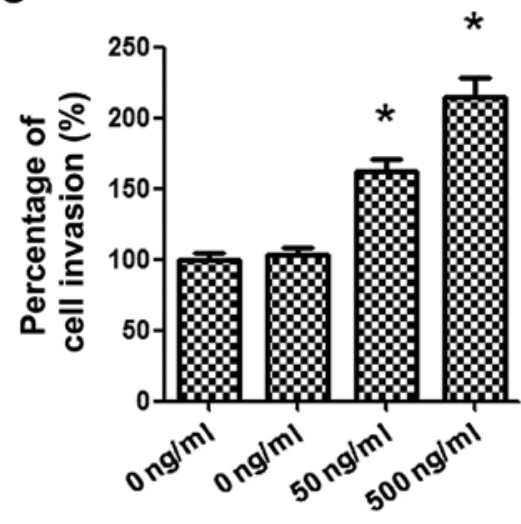

B

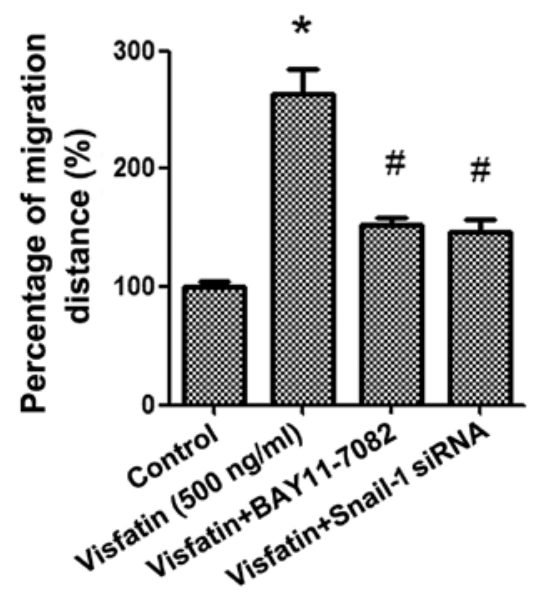

D

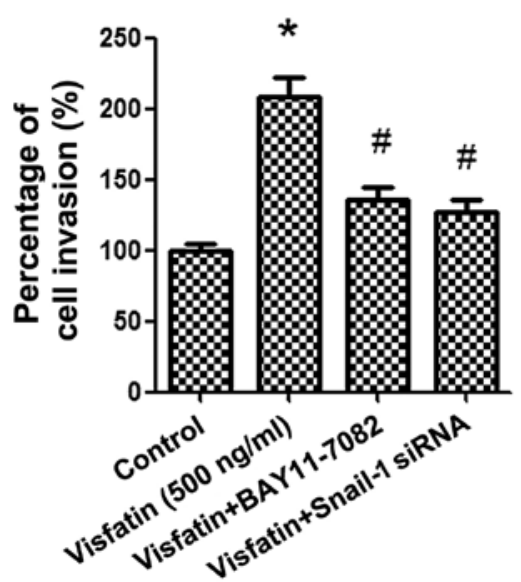

Figure 5. Visfatin enhances the migration and invasion of U2OS osteosarcoma cells. (A and B) U2OS osteosarcoma cells were treated with visfatin at various concentrations $(0,5,50$ and $500 \mathrm{ng} / \mathrm{ml})$ for $24 \mathrm{~h}$. The effect of visfatin on the migration of U2OS osteosarcoma cells was assessed by wound healing assay. (C and D) The effect of visfatin on the invasion of U2OS osteosarcoma cells was assessed by Transwell assay. ${ }^{*} \mathrm{P}<0.05$ vs. the control group; ${ }^{\prime} \mathrm{P}<0.05$ vs. the visfatin group. Data shown are means \pm SEM from three independent experiments in duplicate.

further ascertain the signaling pathway underlying the effects of visfatin on cancer cell migration and invasion, Snail-1 siRNA and BAY11-7082 $(20 \mu \mathrm{M})$ were repectively used to pre-treat the cells for 2 h. As shown in Fig. 5B and D, blockage of the Snail-1 and NF- $\mathrm{B}$ signaling pathways markedly suppressed the migration and invasion of osteosarcoma cells, which suggests that visfatin promotes the migration and invasion of osteosarcoma cells mainly through the Snail-1 and NF- $\mathrm{BB}$ signaling pathways.

\section{Discussion}

Osteosarcoma, arising from primitive transformed cells of mesenchymal origin, mostly occurs in the long bones of children and adolescents, and is the most common malignant tumor of bone (17). The underlying mechanisms involved in the occurrence and development of osteosarcoma are so complex that the exact etiology remains unclear to date. Despite the fact that the mortality rate of osteosarcoma has declined in the past few years, $\sim 30 \%$ of osteosarcoma patients succumb to lung metastases $(18,19)$. As shown in a previous studies, lung is the most common and primary site of metastasis for osteosarcoma (20). Thus, it is urgent to further investigate the potential mechanisms underlying the pathogenesis of osteosarcoma and to search for new factors that affect the migration and invasion of osteosarcoma cells, as well as to explore novel therapeutic approaches and treatment strategies for osteosarcoma patients.

Nampt/PBEF/visfatin has been considered to belong to the dimeric class of type II phosphoribosyltransferases because of its crystal structure $(21,22)$. This enzyme consists of two different forms: an intracellular form, named iNampt and an extracellular form, named eNampt (6). Since little attention has been paid to visfatin, the biological functions and the underlying mechanisms of visfatin remain incompletely understood and its function has been mainly explored in regards to endothelial cells and vascular disorders $(6,23)$. However, along with the findings of its roles in carcinogenesis, recently visfatin has received more attention. As shown in previous studies, visfatin expression is markedly increased in various types of tumors, such as glioblastoma (24), malignant astrocytomas (24), breast $(4,25)$ and prostate cancer (26). In addition, it has been demonstrated that the expression level of visfatin is gradually elevated with the progressive stage of gastric (27) and colorectal cancers (28). Thus, visfatin is regarded as a biomarker for many types of cancer. However, there is still no evidence showing the specific effects of visfatin on osteosarcoma to date, and thus, the present study 
was designed to elucidate the role of visfatin in osteosarcoma, as well as the underlying mechanisms.

Tumor-associated EMT is known as one of the most important contributors promoting osteosarcoma progression and metastasis (17). According to investigations, EMT is associated with elevated grades of incursion, higher tumor recurrence, poorer prognosis and decreased patient survival rates (29). During the process of EMT, the conversion of polarized epithelial cells acquiring a mesenchymal phenotype alters cell-cell and cell-extracellular matrix (ECM) interactions and makes cell motility more unconstrained through the ECM, which is intensively associated with tumor metastasis. The most important marks of EMT are the loss of epithelial marker E-cadherin and the acquisition of mesenchymal marker $\mathrm{N}$-cadherin. Thus, in order to ascertain the effect of visfatin on EMT, the expression levels of E-cadherin and N-cadherin were detected, respectively, at the levels of protein and mRNA following treatment with visfatin. The data (Fig. 1) indicated a significant role of visfatin; vitsfatin treatment concentration- and time-dependently decreased and increased the expression of E-cadherin and $\mathrm{N}$-cadherin, respectively. The results intensively suggest that visfatin is an inducer of EMT in osteosarcoma cells.

Although the molecular mechanisms associated with loss of E-cadherin expression and increase of $\mathrm{N}$-cadherin expression are still not clear, the Snail-1 superfamily, particularly Snail-1, have been considered as the master regulators. A number of experiments have been performed to indicate that Snail-1 is the transcriptional repressor of E-cadherin and transcriptional inducer of N-cadherin. It has been reported that Snail-1 is overexpressed in osteosarcoma (30), and its overexpression inhibits the action of 1,25-dihydroxyvitamin $\mathrm{D}_{3}\left[1,25(\mathrm{OH})-\mathrm{D}_{3}\right]$ which was thought to suppress the proliferation, migration and invasion of cancers $(31,32)$, so that Snail-1 exerts its promoting effect on osteosarcoma progression (30). In the present study, we detected the protein and mRNA expression of Snail-1 after various times of stimulation with visfatin, and the results showed that a 6-h stimulation significantly increased Snail-1 expression. When we blocked Snail-1 with its siRNA, the effects of visfatin on E-cadherin and $\mathrm{N}$-cadherin were significantly reversed, which indicated that visfatin-induced EMT was mediated by Snail-1.

It has been firmly established that $\mathrm{NF}-\kappa \mathrm{B}$ exerts a pivotal role in the regulation of tumor metastasis (33). A previous study proved that the pulmonary metastasis of osteosarcoma is reduced by inhibition of $N F-\kappa B$ (33). In a study of Huber et al (34), it was found that inhibition of $\mathrm{NF}-\kappa \mathrm{B}$ prevented EMT, while activation of this pathway promoted EMT, and inhibition of $\mathrm{NF}-\kappa \mathrm{B}$ caused a reverse in EMT, suggesting that $\mathrm{NF}-\kappa \mathrm{B}$ is essential for EMT. Maier et al (35) and Chen et al (36) also found that $\mathrm{NF}-\kappa \mathrm{B}$ promoted the migration and invasion of carcinoma cells via its induction of EMT. In the present study, we aimed to ascertain whether $\mathrm{NF}-\kappa \mathrm{B}$ was involved in visfatin-induced EMT and the metastasis of osteosarcoma. First, the expression of the p65 subunit of NF- $\kappa \mathrm{B}$ was detected respectively in the nucleus and the cytoplasm, and the increase in the nucleus and the decrease in the cytoplasm indicated the occurrence of $\mathrm{NF}-\kappa \mathrm{B}$ nuclear translocation under the stimulation of visfatin. When the NF- $\kappa$ B pathway was blocked by its inhibitor, the effects of visfatin on the expression levels of E-cadherin and $\mathrm{N}$-cadherin were entirely reversed, as well as the expression of Snail-1. This suggests that NF- $\kappa \mathrm{B}$ is the mediator of visfatin-induced EMT.

In conclusion, the results presented in this study elucidated the visfatin/NF- $\kappa \mathrm{B} / \mathrm{Snail}-1 / \mathrm{EMT}$ pathway as a novel mechanism by which visfatin promotes the aggressive behavior of osteosarcoma cells. In addition, the data above indicate the importance of visfatin as a potential therapeutic target. Thus, inhibition of visfatin may provide a new anti-osteosarcoma strategy.

\section{Acknowledgements}

The present study was supported by the Science Foundation of Shandong Province (no. ZR2014HP005).

\section{References}

1. Link MP, Goorin AM, Miser AW, Green AA, Pratt CB, Belasco JB, Pritchard J, Malpas JS, Baker AR, Kirkpatrick JA, et al: The effect of adjuvant chemotherapy on relapse-free survival in patients with osteosarcoma of the extremity. N Engl J Med 314: 1600-1606, 1986.

2. Luu HH, Zhou L, Haydon RC, Deyrup AT, Montag AG, Huo D, Heck R, Heizmann CW, Peabody TD, Simon MA, et al: Increased expression of S100A6 is associated with decreased metastasis and inhibition of cell migration and anchorage independent growth in human osteosarcoma. Cancer Lett 229: 135-148, 2005.

3. Dalamaga M: Nicotinamide phosphoribosyl-transferase/visfatin: A missing link between overweight/obesity and postmenopausal breast cancer? Potential preventive and therapeutic perspectives and challenges. Med Hypotheses 79: 617-621, 2012.

4. Hufton SE, Moerkerk PT, Brandwijk R, de Bruïne AP, Arends JW and Hoogenboom HR: A profile of differentially expressed genes in primary colorectal cancer using suppression subtractive hybridization. FEBS Lett 463: 77-82, 1999.

5. Folgueira MA, Carraro DM, Brentani H, Patrão DF, Barbosa EM, Netto MM, Caldeira JR, Katayama ML, Soares FA, Oliveira CT, et al: Gene expression profile associated with response to doxorubicin-based therapy in breast cancer. Clin Cancer Res 11: 7434-7443, 2005.

6. Bi TQ and Che XM: Nampt/PBEF/visfatin and cancer. Cancer Biol Ther 10: 119-125, 2010.

7. Hay ED: The mesenchymal cell, its role in the embryo, and the remarkable signaling mechanisms that create it. Dev Dyn 233: 706-720, 2005.

8. Haslehurst AM, Koti M, Dharsee M, Nuin P, Evans K, Geraci J, Childs T, Chen J, Li J, Weberpals J, et al: EMT transcription factors snail and slug directly contribute to cisplatin resistance in ovarian cancer. BMC Cancer 12: 91, 2012.

9. Rosanò L, Cianfrocca R, Spinella F, Di Castro V, Nicotra MR, Lucidi A, Ferrandina G, Natali PG and Bagnato A: Acquisition of chemoresistance and EMT phenotype is linked with activation of the endothelin A receptor pathway in ovarian carcinoma cells. Clin Cancer Res 17: 2350-2360, 2011.

10. Zhang XC, Chen JQ and Li B: Salvianolic acid A suppresses CCL-20 expression in TNF- $\alpha$-treated macrophages and ApoEdeficient mice. J Cardiovasc Pharmacol 64: 318-325, 2014

11. Bae KM, Parker NN, Dai Y, Vieweg J and Siemann DW: E-cadherin plasticity in prostate cancer stem cell invasion. Am J Cancer Res 1: 71-84, 2011.

12. Pon YL, Auersperg N and Wong AS: Gonadotropins regulate $\mathrm{N}$-cadherin-mediated human ovarian surface epithelial cell survival at both post-translational and transcriptional levels through a cyclic AMP/protein kinase A pathway. J Biol Chem 280: 15438-15448, 2005.

13. Li B, Dong Z, Liu H, Xia YF, Liu XM, Luo BB, Wang WK, Li B, Gao F, Zhang C, et al: Serum amyloid A stimulates lipoproteinassociated phospholipase A2 expression in vitro and in vivo. Atherosclerosis 228: 370-379, 2013.

14. Peinado H, Olmeda D and Cano A: Snail, Zeb and bHLH factors in tumour progression: An alliance against the epithelial phenotype? Nat Rev Cancer 7: 415-428, 2007. 
15. Zhao Z, Wu MS, Zou C, Tang Q, Lu J, Liu D, Wu Y, Yin J, Xie X, Shen J, et al: Downregulation of MCT1 inhibits tumor growth, metastasis and enhances chemotherapeutic efficacy in osteosarcoma through regulation of the NF- $\kappa \mathrm{B}$ pathway. Cancer Lett 342 : $150-158,2014$.

16. Li Y, Zhang ZN, Zhao HM, Tong ZC, Yang J, Wang H and Liang XJ: Matrine inhibits the invasive properties of human osteosarcoma cells by downregulating the ERK-NF- $\kappa \mathrm{B}$ pathway. Anticancer Drugs 25: 1035-1043, 2014.

17. Yang G, Yuan J and Li K: EMT transcription factors: Implication in osteosarcoma. Med Oncol 30: 697, 2013.

18. PosthumaDeBoer J, Witlox MA, Kaspers GJ and van Royen BJ: Molecular alterations as target for therapy in metastatic osteosarcoma: A review of literature. Clin Exp Metastasis 28: 493-503, 2011.

19. Krishnan K, Khanna $\mathrm{C}$ and Helman LJ: The biology of metastases in pediatric sarcomas. Cancer J 11: 306-313, 2005.

20. Harting MT and Blakely ML: Management of osteosarcoma pulmonary metastases. Semin Pediatr Surg 15: 25-29, 2006.

21. Khan JA, Tao X and Tong L: Molecular basis for the inhibition of human NMPRTase, a novel target for anticancer agents. Nat Struct Mol Biol 13: 582-588, 2006.

22. Wang T, Zhang $X$, Bheda P, Revollo JR, Imai S and Wolberger $C$ : Structure of Nampt/PBEF/visfatin, a mammalian NAD ${ }^{+}$biosynthetic enzyme. Nat Struct Mol Biol 13: 661-662, 2006.

23. Kim SR, Bae SK, Choi KS, Park SY, Jun HO, Lee JY, Jang HO, Yun I, Yoon KH, Kim YJ, et al: Visfatin promotes angiogenesis by activation of extracellular signal-regulated kinase $1 / 2$ Biochem Biophys Res Commun 357: 150-156, 2007.

24. Reddy PS, Umesh S, Thota B, Tandon A, Pandey P, Hegde AS, Balasubramaniam A, Chandramouli BA, Santosh V, Rao MR, et al: PBEF1/NAmPRTase/Visfatin: A potential malignant astrocytoma/glioblastoma serum marker with prognostic value. Cancer Biol Ther 7: 663-668, 2008.

25. Van Beijnum JR, Moerkerk PT, Gerbers AJ, De Bruïne AP, Arends JW, Hoogenboom HR and Hufton SE: Target validation for genomics using peptide-specific phage antibodies: A study of five gene products overexpressed in colorectal cancer. Int J Cancer 101: 118-127, 2002.

26. Patel ST, Mistry T, Brown JE, Digby JE, Adya R, Desai KM and Randeva HS: A novel role for the adipokine visfatin/pre-B cell colony-enhancing factor 1 in prostate carcinogenesis. Peptides 31: 51-57, 2010.
27. Nakajima TE, Yamada Y, Hamano T, Furuta K, Gotoda T, Katai H, Kato K, Hamaguchi T and Shimada Y: Adipocytokine levels in gastric cancer patients: Resistin and visfatin as biomarkers of gastric cancer. J Gastroenterol 44: 685-690, 2009.

28. Nakajima TE, Yamada Y, Hamano T, Furuta K, Matsuda T, Fujita S, Kato K, Hamaguchi T and Shimada Y: Adipocytokines as new promising markers of colorectal tumors: Adiponectin for colorectal adenoma, and resistin and visfatin for colorectal cancer. Cancer Sci 101: 1286-1291, 2010.

29. Cichon MA and Radisky DC: ROS-induced epithelial-mesenchymal transition in mammary epithelial cells is mediated by $\mathrm{NF}-\kappa \mathrm{B}-$ dependent activation of Snail. Oncotarget 5: 2827-2838, 2014.

30. Yang H, Zhang Y, Zhou Z, Jiang X and Shen A: Snail-1 regulates VDR signaling and inhibits $1,25(\mathrm{OH})-\mathrm{D}_{3}$ action in osteosarcoma. Eur J Pharmacol 670: 341-346, 2011.

31. Larriba MJ, Ordóñez-Morán P, Chicote I, Martín-Fernández G, Puig I, Muñoz A and Pálmer HG: Vitamin D receptor deficiency enhances Wnt/ $\beta$-catenin signaling and tumor burden in colon cancer. PLoS One 6: e23524, 2011.

32. Stubbins RE, Hakeem A and Núñez NP: Using components of the vitamin D pathway to prevent and treat colon cancer. Nutr Rev 70: 721-729, 2012

33. Nishimura A, Akeda K, Matsubara T, Kusuzaki K, Matsumine A, Masuda K, Gemba T, Uchida A and Sudo A: Transfection of NF- $\kappa \mathrm{B}$ decoy oligodeoxynucleotide suppresses pulmonary metastasis by murine osteosarcoma. Cancer Gene Ther 18: 250-259, 2011.

34. Huber MA, Azoitei N, Baumann B, Grünert S, Sommer A, Pehamberger H, Kraut N, Beug H and Wirth T: NF-kappaB is essential for epithelial-mesenchymal transition and metastasis in a model of breast cancer progression. J Clin Invest 114: 569-581, 2004.

35. Maier HJ, Schmidt-Strassburger U, Huber MA, Wiedemann EM, Beug $\mathrm{H}$ and Wirth T: NF-kappaB promotes epithelialmesenchymal transition, migration and invasion of pancreatic carcinoma cells. Cancer Lett 295: 214-228, 2010.

36. Cheng ZX, Sun B, Wang SJ, Gao Y, Zhang YM, Zhou HX, Jia G, Wang YW, Kong R, Pan SH, et al: Nuclear factor- $\kappa \mathrm{B}$-dependent epithelial to mesenchymal transition induced by HIF-1 $\alpha$ activation in pancreatic cancer cells under hypoxic conditions. PLoS One 6: e23752, 2011. 